LA-UR- $93-\mathbf{3} 884$

Title:
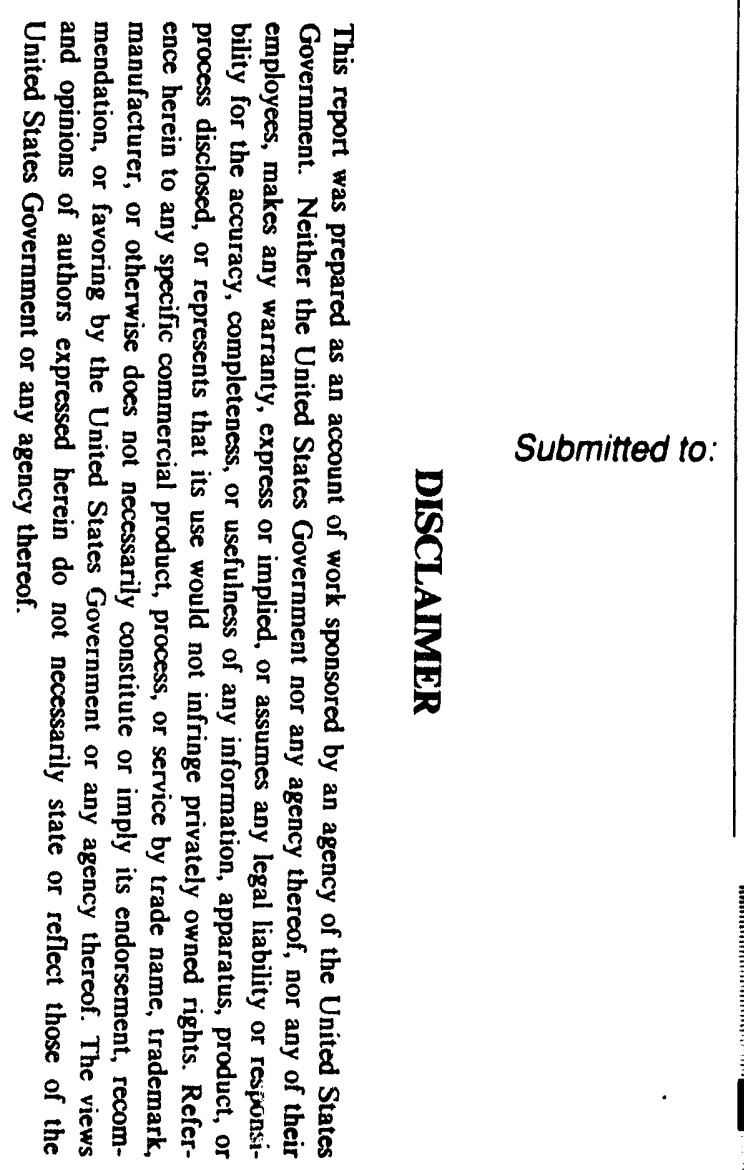

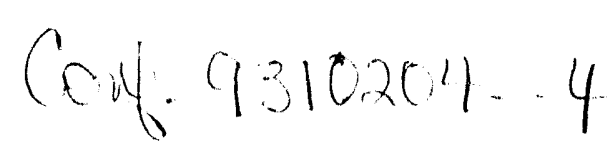

Beam Diagnostics at High-Intensity Storage Rings
Beam Instrumentation Workshop

Santa Fe, New Mexico, October 20-23, 1993

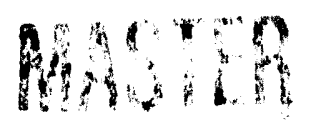

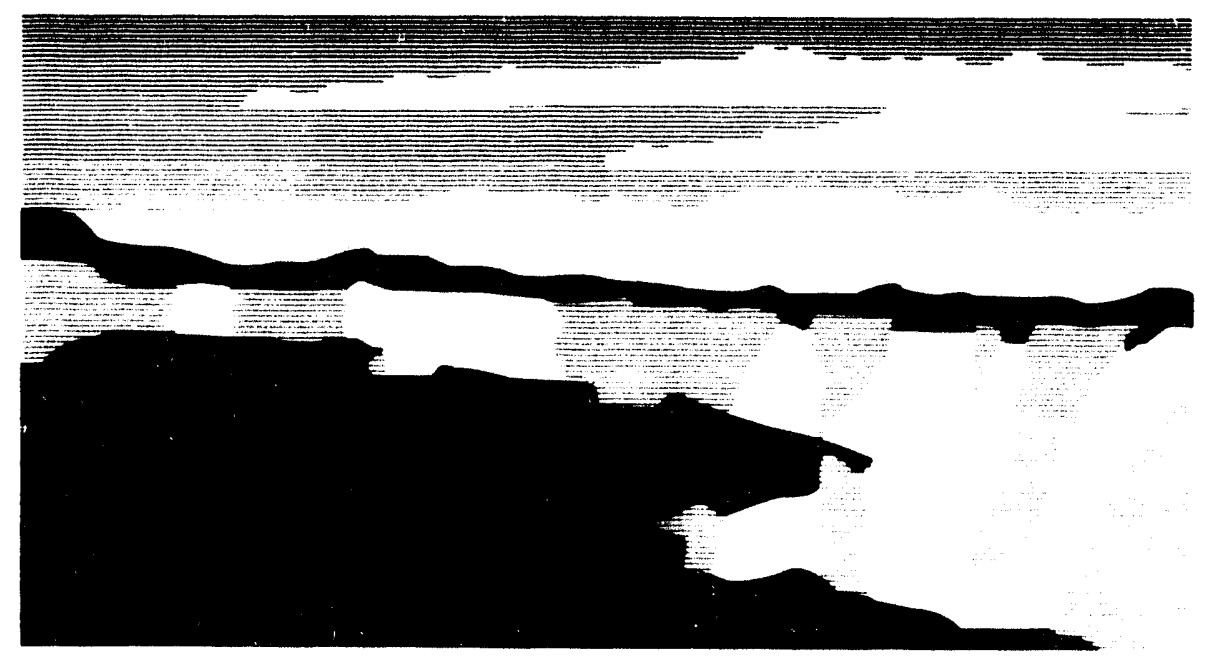

Los Alamos National Laboratory, an aftirmative action/equal opportunity employer, is operated by the University of California for the U.S. Department of Energy under contract W.7405-ENG-36. By acceptance of this article, the publisher recognizes that the U.S. Government retains a nonexclusive, royalty-tree license to publish or reproduce the published form of this contribution, or to allow others to do so, for U.S. Government purposes. The Los Alamos National Laboratory requests that the publisher identify this article as work perfor! ned under the auspices of the U.S. Department of Energy. 


\title{
BEAM DIAGNOSTICS AT HIGH-INTENSITY STORAGE RINGS
}

\author{
Mike Plum \\ MP-5, Los Alamos National Laboratory, Los Alamos, NM 87545
}

\begin{abstract}
Beam diagnostics at high-intensity facilities feature their own special set of problems and characteristics. Issues peculiar to high-intensity storage rings include beam loss, beam halos, extraction efficiency, beam in the gap, clearing electrodes, and beam-profile measurement. The Los Alamos Proton Storage Ring (PSR) is a nice example of a highintensity storage ring. I will discuss in some detail three diagnostic systems currently in use at the PSR: the beam-loss-monitor system, the electron-clearing system, and the beam-inthe-gap monitor. Much of our discussion is inspired by the problems we have encountered and the useful things we have learned while commissioning and developing the PSR. Another inspiration is our work on the next-generation neutron-spallation source, also known as the National Center for Neutron Research (NCNR).
\end{abstract}

\section{INTRODUCTION}

Beam diagnostics at high-intensity facilities present their own special set of problems and concerns. Some of the issues pecullar to high-intensity storage rings are beam loss, beam halos, extraction efficiency, beam in the gap, clearing electrodes, and beam-profile measurement. Perhaps the most important of these is beam loss. It may be perfectly acceptable to lose a percent or more of the beam in some machines, especially in the transport lines and during the injection and extraction processes. However, in high-intensity machines, losing just one percent of the beam can cause unacceptable damage to beam-line components through thermal heating and radiation damage. Beam loss can also make hands-on maintenance difficult or impossible because of high residual radioactivity levels. At the Los Alamos Meson Physics Facility (LAMPF) we have the additional complication that beam loss can cause prompt radlation in occupied areas. This is because our passive shielding is not up to the task if we consider the worst-case scenarios of losing the maximum possible beam current where the shielding is the weakest. A good real-time beam-loss system is therefore very important, especially since in practice we always end up fine-tuning the facility to minimize the beam loss.

Beam loss during extraction is similarly important, since it is not acceptable to lose even one percent of the beam during the extraction process. It is therefore important to have an efficient extraction process, and to be able to measure the extraction efficiency. For example, at the Proton Storage Ring (PSR) we limit extraction losses to about $0.2 \%$, but even this level will be too high for the next-generation neutron-spallation sources.

It is important to maintain a beam-free gap in the circulating beam for extraction efficiency and beam instability. At the PSR, beam in the gap may lead to electron trapping, and electrons are a likely source of our instability. It is difficult to measure how much beam is in the gap because we are interested in intensity levels about four orders of magnitude below the peak beam intensity, and the time structure is on the scale of tens of nanoseconds.

Beam halo is another important ingredient in the beam-loss formula, since beam halos scrape off at aperture restrictions and cause beam loss. Halo formation is a complicated phenomenon that is not well understood. ${ }^{1}$ Halos are also difficult to measure, since one needs profile monitors that can accurately measure beam tails four orders of magnitude below the peak beam intensity. This is why good beam-profile monitors are important at 
high-intensity facilities. High-intensity machines offer the additional challenge of overcoming space-charge effects, which, for example, can make residual-gas profile monitors impractical.

To deal with the electrons and electron trapping, we need to consider clearing electrodes. High-intensity facilities lend an additional complication to clearing-electrode systems since we must bias the electrodes with potentials high enough to create electric fields that will overcome the field of the beam. For example, at the PSR we need about $20 \mathrm{kV}$ on our clearing electrodes.

Much of what I plan to discuss is inspired by the problems we have encountered and the useful things we have learned while commissioning and developing the PSR. Another inspiration is our work on the next-generation neutron-spallation source, also known as the National Center for Neutrun Research (NCNR). I will describe three diagnostic systems presently in use at the PSR as real-life examples that involve high-intensity issues .- the loss-monitor system, the electron-clearing system, and the beam-in-the-gap monitor. Since I'm going to talk a lot about the PSR, allow me to introduce you to the LAMPF/PSR facility. A diagram of the facility is shown in Fig. 1.

\section{THE LOS ALAMOS PROTON STORAGE RING}

The purpose of the Proton Storage Ring (PSR) is to compress in time the 800-MeV proton beam from the LAMPF linac. The beam is first divided into a series of 250-ns-long bunches at 360-ns intervals by the beam chopper in the low-energy transport region of the linac. These bunches are then accelerated to $800 \mathrm{MeV}$ and injected into the PSR, which has a revolution period of $360 \mathrm{~ns}$. The bunches are therefore stacked one atop the other, to create a single 250-ns bunch with the same charge as the injected $600-\mu$ s bunch, for a compression factor of about 2400 . The beam is then delivered to a neutron-spallation target, where a short burst of neutrons is generated for materials-science, biology, and nuclearphysics experiments. It is necessary for the neutron burst to be short so time-of-flight techniques may be used to measure the neutron energies. Some of the more important PSR parameters are shown in Table 1, and Table 2 compares our achieved pertormance to our design goals.

Table 1. A list of some PSR parameters.

\begin{tabular}{|l|l|}
\hline Proton kinetio energy & $797 \mathrm{MeV}$ \\
\hline Circumference & $90.2 \mathrm{~m}$ \\
\hline Focusing structure & FODO, 10 cell \\
\hline Betatron tunes $Q_{x,} Q_{Y}$ & $3.23,2.22$ \\
\hline Acceptance $A_{x,} A_{Y}$ & $124,167 \mathrm{~mm}-\mathrm{mrad}$ \\
\hline Transition gamma & 3.1 \\
\hline Average pipe radius & $0.05 \mathrm{~m}$ \\
\hline Measured chromaticities $\xi_{x,} \xi_{y}$ & $-1.3,-1.0$ \\
\hline Buncher harmonic, frequency & $1,2.795 \mathrm{MHz}$ \\
\hline Peak nt voltage & $15 \mathrm{kV}$ \\
\hline Maximum synchrotron tune $\mathrm{Q}_{s}$ & 0.0006 \\
\hline Bunch length & $60 \mathrm{~m}$ \\
\hline Repetition rate & $20 \mathrm{~Hz}$ \\
\hline
\end{tabular}




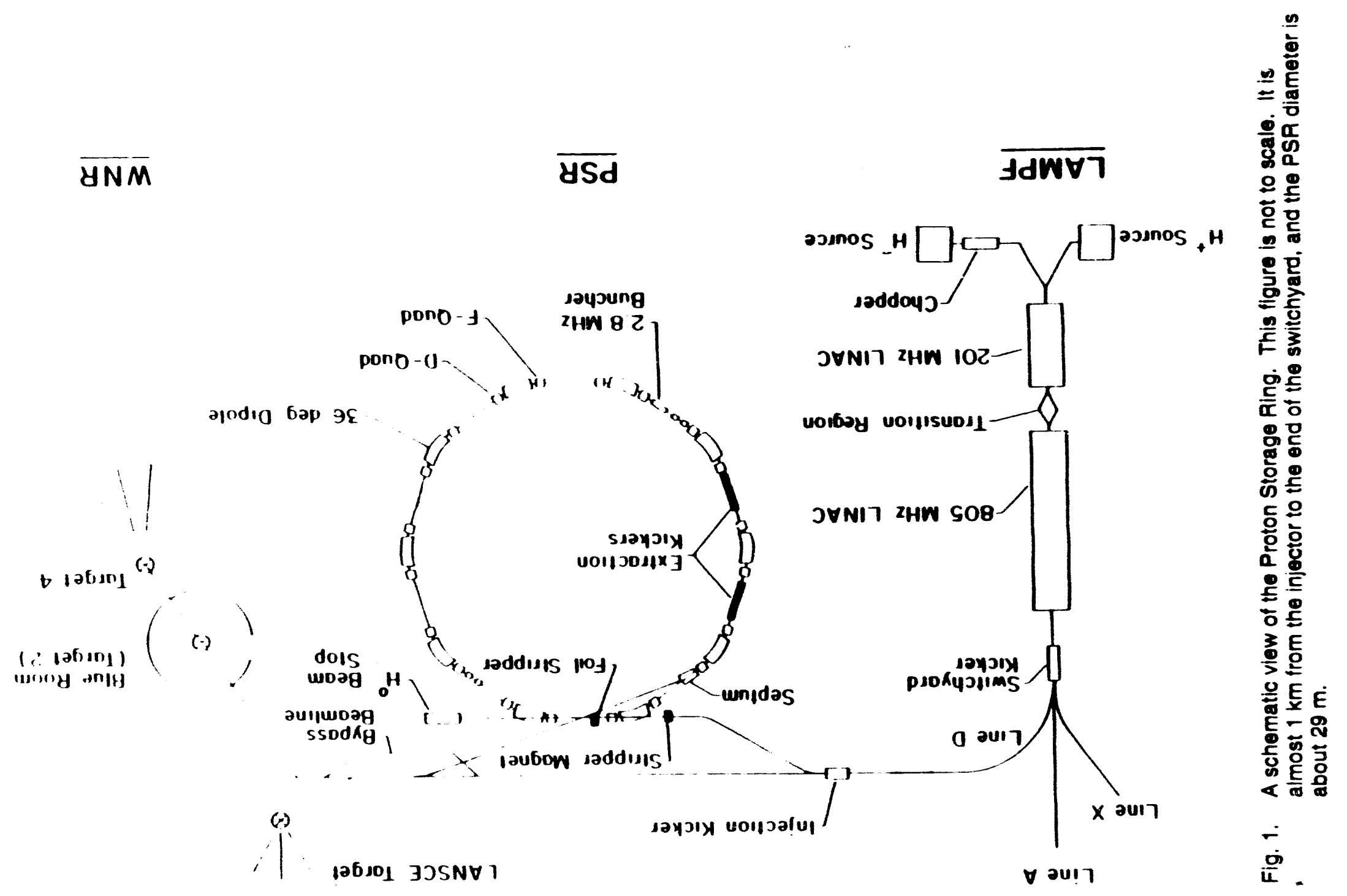


Table 2. A comparison of design and achieved goals.

\begin{tabular}{|l|l|l|}
\hline & Design & Achieved \\
\hline Peak injected current & $15 \mathrm{~mA}$ & $10-11 \mathrm{~mA}$ \\
\hline Injection pulse length & $750 \mu \mathrm{s}$ & $975 \mu \mathrm{s}$ \\
\hline Protons/pulse & $5.2 \times 10^{13}$ & $3.8 \times 10^{13}$ \\
\hline Repetition rate & $12 \mathrm{~Hz}$ & $20 \mathrm{~Hz}$ \\
\hline Average current & $100 \mu \mathrm{A}$ & $75 \cdot 80 \mu \mathrm{A}$ \\
\hline Losses: & & \\
\hline Accumulation & 0.1 to $0.3 \%$ & $0.6100 .7 \%$ \\
\hline Extraction & $<0.1 \%$ & 0.1 to $0.3 \%$ \\
\hline
\end{tabular}

The PSR employs a unique two-step injection process. The incoming $\mathrm{H}^{*}$ ion beam is first stripped to $\mathrm{H}^{\circ}$ by a high-field stripper magnet located just upstream of the first magnet in the ring. This neutral beam, unaffected by magnetic fields, drifts through a hole in the first dipole magnet of the ring and begins traveling along the path of the circulating protons in the ring. The $\mathrm{H}^{\circ}$ particles then pass through a thin $200-\mu \mathrm{g}$ stripper foil where they are converted to $\mathrm{H}^{+}$, and they begin to circulate around the ring along with the other $\mathrm{H}^{+}$particles. The $\mathrm{H}^{+}$ particles already in the ring also pass through the stripper foll, where they are unaffected except for a slight amount of emittance growth caused by Coulomb and nuclear scattering.

Let us now focus in on three systems presently in use at the PSR that involve high. intensity issues: the loss-monitor system, the electron-clearing system, and the beam-in-thegap monitor. The latter two systems were installed earlier this year.

\section{THE PSR LOSS-MONITOR SYSTEM}

At the PSR the average beam current is about $70 \mu \mathrm{A}$ and the peak current is about $28 \mathrm{~A}$. For such a machine one would like to keep the beam loss below 1 part in $10^{4}$, to allow hands-on maintenance of the machine and to prevent damage to beam-line components. However, the high level of beam loss at the PSR prevents us from reaching our design goal of $100 \mu \mathrm{A}$, and so we allow an average of $0.5 \mu \mathrm{A}$ of beam loss, which is about $0.7 \%$ of the average beam current. Our residual activation levels routinely reach as high as $20 \mathrm{rad} / \mathrm{h}$ on contact, which is as high as we can tolerate. Beam loss is a very important issue for us, and we constantly run on the edge of tolerable losses.

To measure the PSR beam loss we use photomultiplier tubes immersed in 0.5 -liter cans filled with scintillating fluid. An electronics module converts the average current to a voltage, which is read out by the controls computer. Twenty of these detectors are located on the beam-tunnel walls, equally spaced around the perimeter of the ring, at beam-line height, as shown in Fig. 2. Once each year the detectors are first gain balanced by collecting the detectors in groups of five, placing the group alongside a reference can, spiiling beam to illuminate that location, and then adjusting the high voltages to equalize the gain of each can.

The detectors are then redistributed around the ring, and a known amount of beam is spilled in the ring by turning off the extraction kickers and allowing all of the injected beam to be lost (the ilfetime is only about $10 \mathrm{~ms}$ ). The pattern of the beam spill is then varied by putting bumps in the ring orbit. By measuring the summed signal from all twenty detectors as a function of the beam-spill pattern, we can show that the summed signal is mostly independent of the nature of the beam spill. The summed signal therefore gives us an absolute measurement of the amount of beam spilled in the ring. With this system we are able to accurately measure beam spill of a fraction of a percent, no matter where or how the beam is lost in the ring, and we can also determine the approximate origin of the spllis. We 


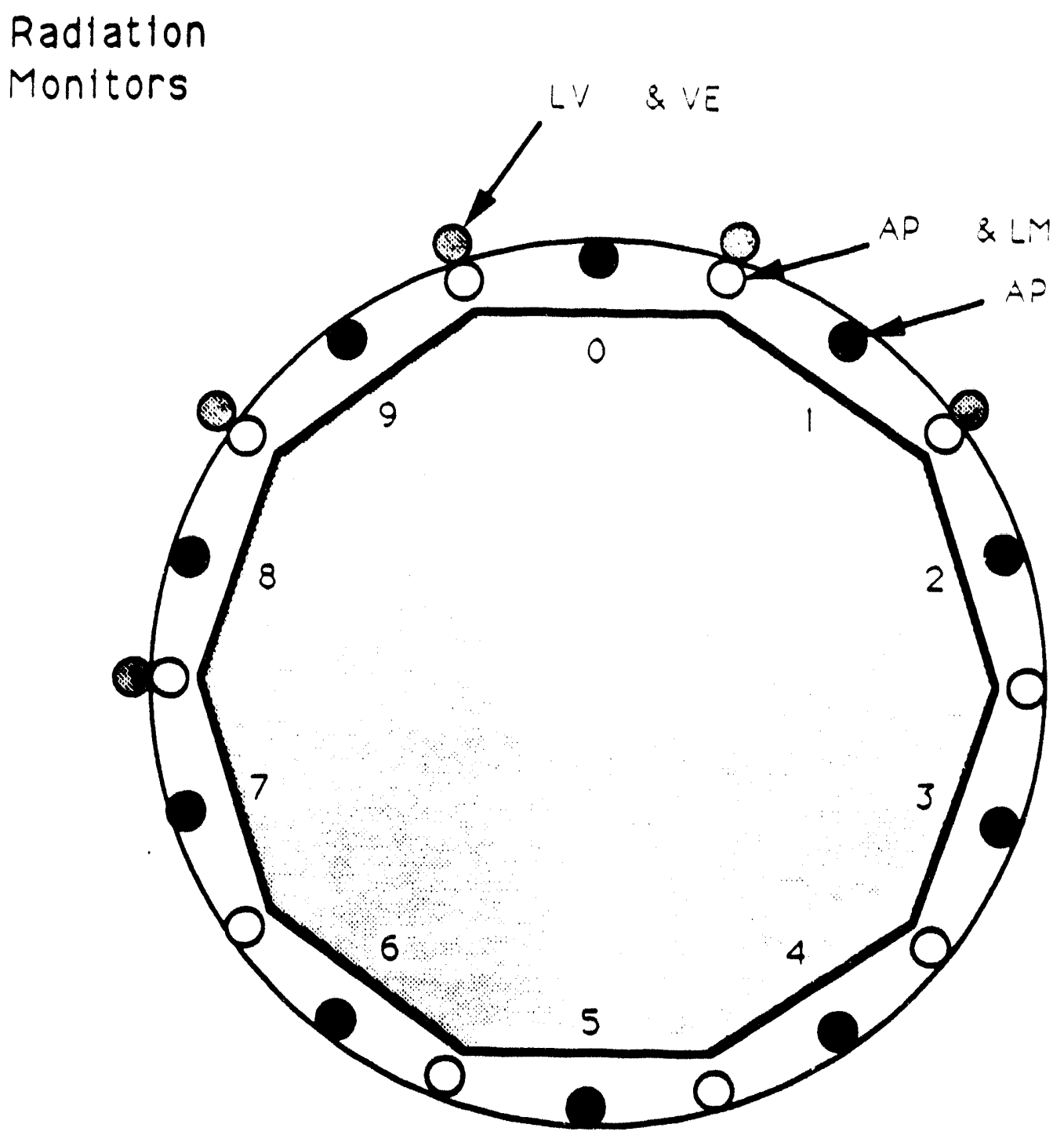

Fig. 2. Loss monitor locations in the PSA. The APs are nine-stage photomultipller tubes immersed in 0.5-liter cans of scintillating fluid, connected to averaging electronics. The LMs are the same as the APs but the analog signals are available for viewing in the control room. The LVs are the same as the LMs but the last four stages of amplification are shorted to avoid saturating on extraction losses, and the VEs are vacuum photodiodes, also for measuring extraction losses. 
estimate the error in the measurement to be about $30 \%$. An example of a recent calibration is shown in Fig. 3. Some of the more relevant specifications are shown in Table 3.

Table 3. Some specifications of the PSR loss-monitor system.

\begin{tabular}{|l|l|}
\hline Absolute accuracy of total ring loss & $-30 \%$ \\
\hline Phototube & RCA 4552 \\
\hline Scintillator fluid & $1 \mathrm{pt}$ NE235H \\
\hline Time to shut off beam & $-30 \mu \mathrm{s}$ \\
\hline Dynamic range & $10^{5}$ \\
\hline
\end{tabular}

Some disadvantages of a photomultiplier-tube-based system are that each tube needs a separate high-voltage supply, gains can change with age, and if a defective tube must be replaced, the new tube must first be calibrated. Scintillator fluid is also a hazardous substance, and when irradiated it becomes a mixed waste. The nice thing about photomultipliers is they are fast. If speed is not critical then ion chambers can be a good choice. We are presently in the process of upgrading our photomultiplier-tube-based system to an ion-chamber-based system.

Earlier I mentioned that the passive shielding at LAMPF is not adequate to protect against the worst-case beam-spill scenarios. We therefore embarked on an extensive effort to build a system of loss monitors and radiation detectors qualified for use in a personal safety system. We settled on a three-prong approach: a fall-safe lon-chamber-based system located in the beam tunnels to detect beam spill, ${ }^{2}$ current limiting toroids located in the beam lines to detect excess beam current, ${ }^{3}$ and neutron detectors located in the occupied areas to detect unsafe levels of prompt radlation due to beam spill. ${ }^{4}$ These systems shut off the beam if they detect unsafe operating conditions. LAMPF is the only facility that I know of, probably the only one in the world, that relles so extensively on electronic systems for this purpose. I would like to discuss the fail-safe ion-chamber system as an example of how a loss-monitor system can be used for personal protection.

For the fail-safe ion-chamber system we use ion chambers filled with nitrogen gas at $1 \mathrm{std}$. atm., so if the lon chamber springs a leak, and the lon chamber becomes filled with air at local pressure $\left(0.80 \mathrm{std}\right.$. atm.), the ion-chamber response will change by just $20 \%{ }^{5} \mathrm{~A}$ $20 \%$ change is big enough to detect with a radioactive sou"ce, but small enough that it will not compromise personnel safety. We also use a 40-G $\Omega$ resistor between the high-voltage and signal electrodes to generate a background current to check that the high voltage is set to its proper value, all the cables are connected, and to check the internal connections within the ion chamber. We have spent several man-years developing the electronics to be failsafe against loss of power, any fallure of any single component, and any two points in the circuit becoming connected by any resistance. The latest generation of the system has been performing nicely since it was first installed in 1991. Some of the more relevant specifications are shown in Table 4, and Fig. 4 shows a schematic view of the lon chamber.

Table 4. Some specifications for the fall-safe lon-chamber system.

\begin{tabular}{|l|l|}
\hline Response time & $-50 \mathrm{~ms}$ \\
\hline Ion chamber actlve volume & $180 \mathrm{~cm}^{3}$ \\
\hline lon chamber gas & Nitrogen at 1 std. atm. \\
\hline Threshold range & 8108000 rads $/ \mathrm{h}$ \\
\hline Dynamic range of detectable signals & $-5 \times 10^{4}$ \\
\hline
\end{tabular}

I chose to discuss the fail-safe lon-chamber system as an example of how one can use active electronics for personnel protection, and because the need for such a system will be greatest at high-intensity facilities. However, I should point out that it is far better to use 
PSR loss-monitor callbration on July 13, 1993

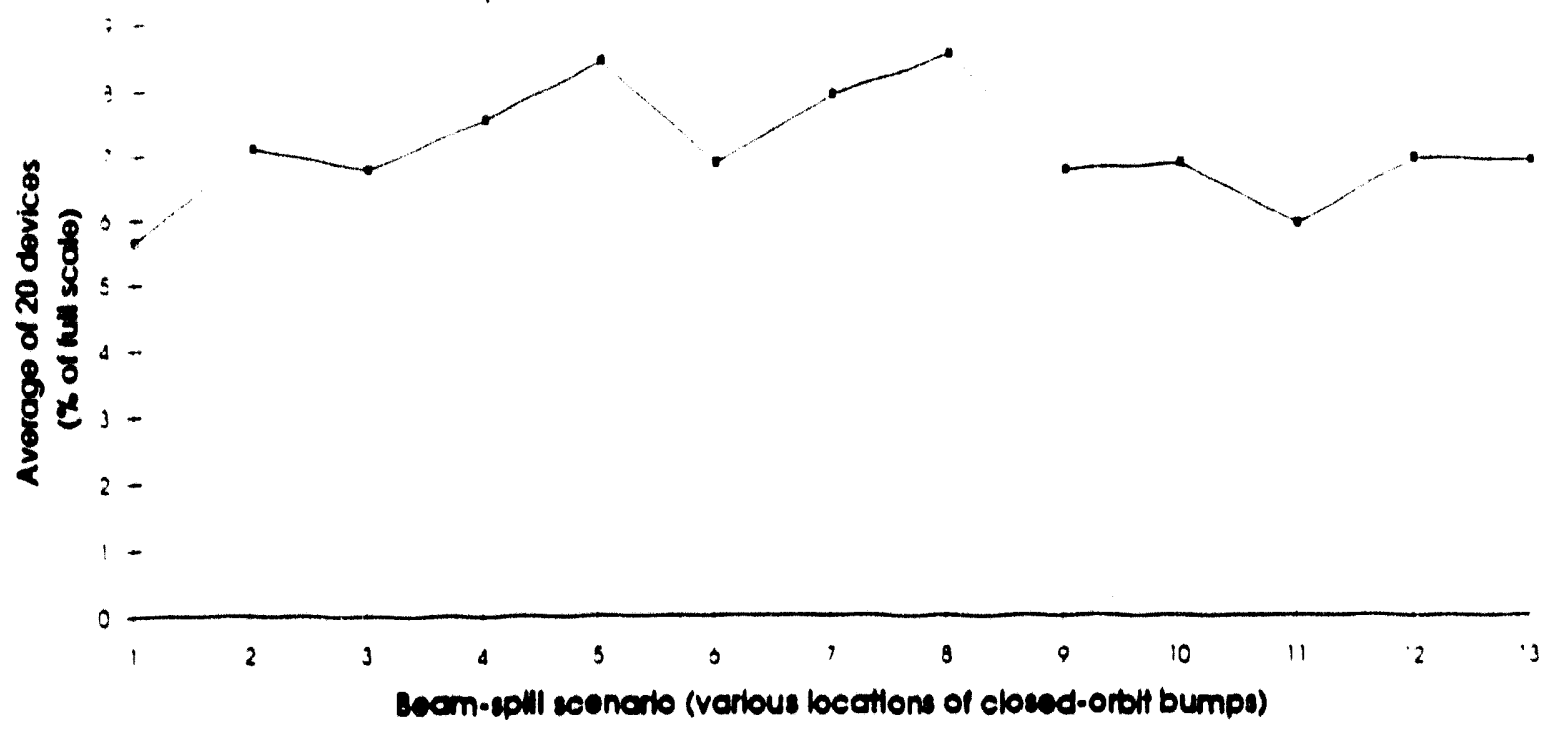

Fig. 3. An example of how the perimeter loss-monitor system responds to a constant beam spill for various beam spill scenarnos, created by putting closed-orbit bumps in different locations in the ring.

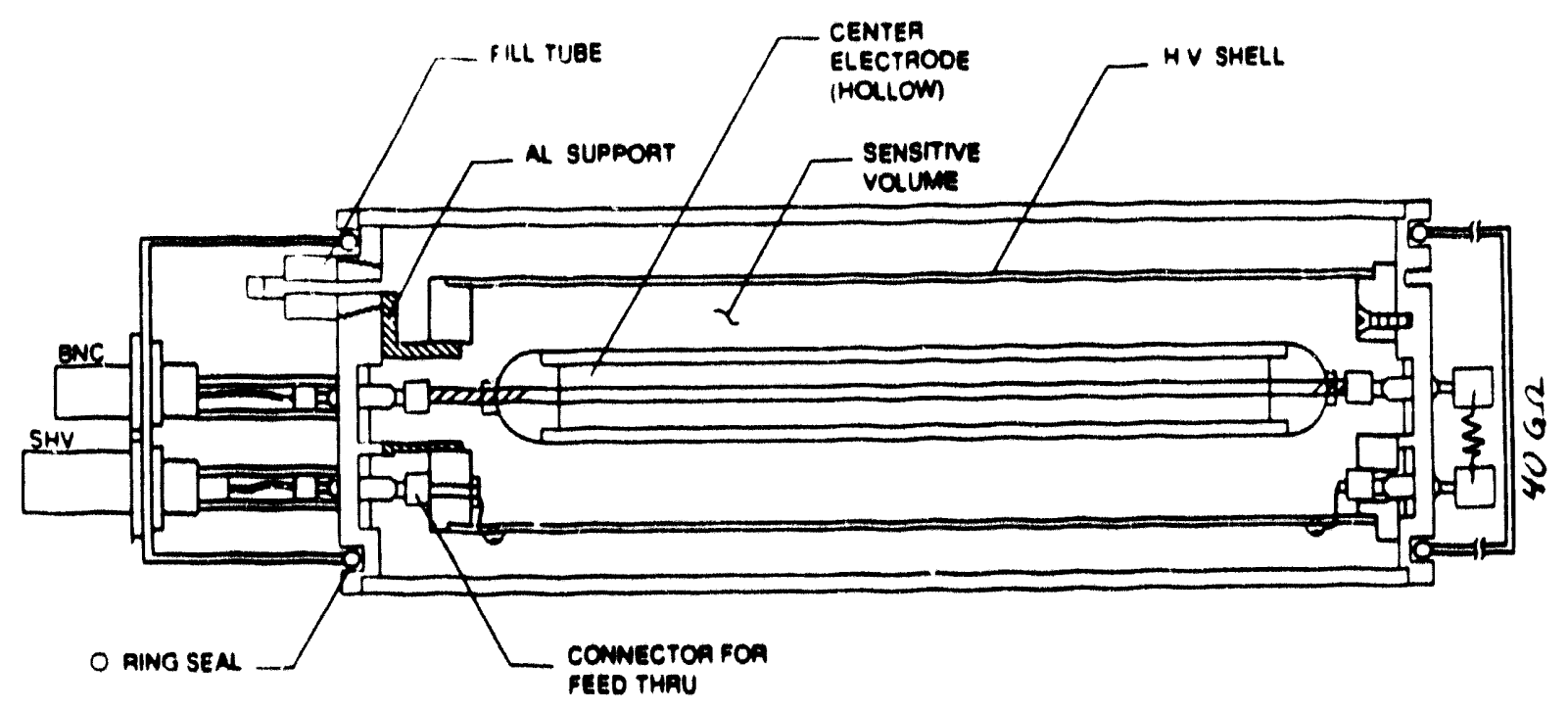

Fig. 4. The ion chamber used in the fail-safe ion-chamber system. 
sufficient passive shielding instead. It is safer, and one can install it and forget it. Electronic systems require constant maintenance and checking, and in the long run they do not pay off.

\section{THE PSR ELECTRON-CLEARING SYSTEM}

Let us now move on to the electron-clearing system. Electrons are an important issue for the PSR because we suspect our instability is an e.p instability. In this instability, background low-energy electrons are trapped in the space-charge potential of the proton beam. Above a certain threshold the electrons and protons develop coupled oscillations that grow in time, leading eventually to beam loss. ${ }^{6}$ The biggest source of electrons at the PSR is in the injection section, also known as section 0 . In this section we have secondary emission (SEM) and thermionic emission of electrons from the stripper foil, residual-gas ionization, beam scraping on the beam-pipe walls causing further SEM of electrons, and electrons stripped from the injected $H^{\circ}$ particles, also known as convoy electrons. We also have delta-ray electrons, with energies up to $2.4 \mathrm{MeV},{ }^{7}$ caused by knock-on collisions of the protons with electrons in the foil.

We built three subsystems to clear out these electrons: 1) clearing electrodes for the ions and electrons from residual gas ionization, and the electrons from SEM off the beampipe walls caused by beam-halo scraping; 2 ) a bending magnet and Faraday cup for the convoy electrons; and 3) a stripper-foil-biasing system to prevent the thermionic and SEM electrons from leaving the foll. The system is illustrated in Fig. 5.

The clearing electrodes work by creating an electric fleld (E-fleld) strong enough to overcome the E-field due to the beam. In high-intensity machines, these space-charge fields can be considerable. For example, from a case taken from the PSA, for a Gaussian beam with $\sigma_{x}=3.4 \mathrm{~mm}, \sigma_{y}=8.9 \mathrm{~mm}$, and a beam current of $43 \mathrm{~A}$, the maximum space-charge electric fleld is about $250,000 \mathrm{~V} / \mathrm{m}$. This translates to about $+1-20 \mathrm{kV}$ on the clearing electrodes. Note that the space-charge fields will be even greater for the next-generation neutron-spallation source. In general it may be necessary to put clearing electrodes in every cell of the machine, and if this is the case one must also consider the effects of coupling impedance. We installed two pairs of clearing electrodes, one upstream and one downstream of the stripper foil. Our electrodes are curved to match the cross section of the beam pipe, and each one covers 90 degrees of the circumference. Each electrode can be biased up to $30 \mathrm{kV}$.

The purpose of the Faraday cup is to clear out the $430-k e V$ convoy electrons. The energy of these electrons is too high to clear with electrodes, so we chose to bend them into a Faraday cup with a low-field bending magnet. To install the device we inserted a 6 -inch tee into the beam line as close as possible to the stripper foll. The first of two C-magnets bends the convoy electrons 90 degrees into the Faraday cup, which fills as much of the 6 inch pipe as possible. The second magnet is identical to but opposite in polarity from the first. It is located just downstream of the first to cancel the closed-orbit distortion and the nonlinear magnetic fields. We also installed a screen at the entrance to the Faraday cup, flush with the inside of the beam pipe, to avoid any wake-field effects. We can blas this screen to reduce the SEM effects when the convoy electrons hit the Faraday cup, and also to attract any low-energy electrons and negative lons from within the beam-plpe volume. Blasing the Faraday cup to $200 \mathrm{~V}$ and the foll to $100 \mathrm{~V}$ is more than sufficient to clear the convoy electrons.

To deal with the SEM and thermionic electroris from the foll we cannot simply use clearing electrodes or clearing rings. The E-field would be strong between the foll frame and the electrodes but weak at the center of the foll where we need it the most. So we chose to blas the stripper foll. With our high beam intensities the electric fleld at the center of the surface of the foil is about $1 \mathrm{MV} / \mathrm{m}$, assuming a perfectly conducting foll. Because of the very high surface E-field created by the beam we cannot bias the foll enough to prevent the SEM and thermionic electrons from being ripped off. However, we can create a potential well a centimeter or so away from the foll if we bias it below the depth of the beam's potential 


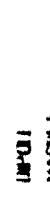

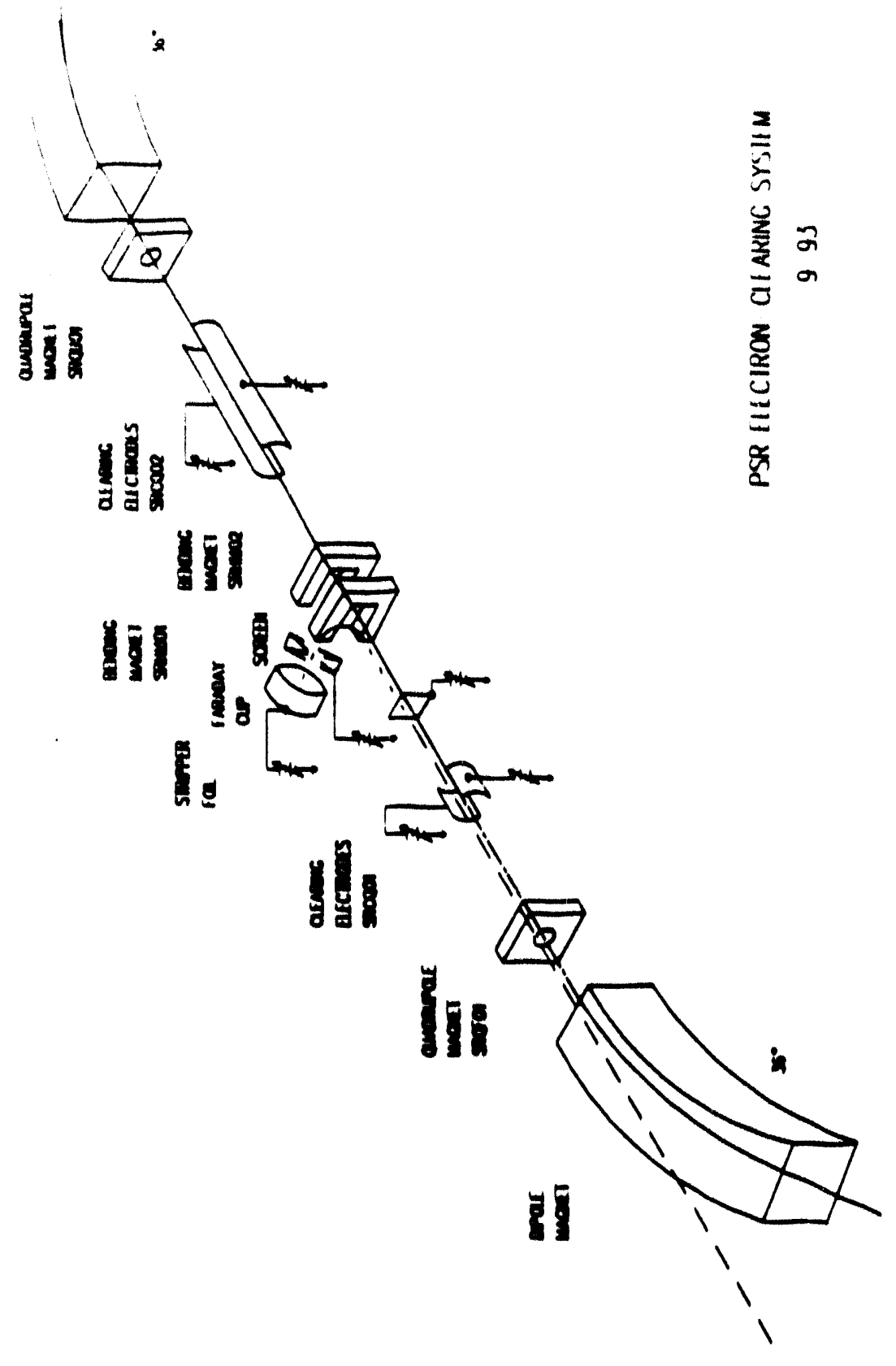

Fig. 5. The PSR Electron-Clearing System. The solid line represents the circulating proton beam, the dashed line repiseents the incoming $\mathrm{H}^{\mathrm{O}}$ beam, and the dotted line represents the electrons stripped from the $\mathrm{H}^{\circ}$ beam. 
well. The electrons will then be trapped until the beam intensity drops at the end of the bunch, when the bias E-field will be strong enough to attract the electrons so they can be absorbed on the foil surface. The electrons are therefore cleared after each revolution of the beam around the ring, or about every $360 \mathrm{~ns}$. The potentlal well criated by the beam is about $8 \mathrm{kV}$ deep, and we can blas the foil up to about $10 \mathrm{kV}$, llmited by the existing construction of the foll-mounting system.

These three subsystems deal efficiently with all the electron sources but one - the delta-ray electrons. These electron energies span the range from a few eV to $2.4 \mathrm{MeV}$. Most of the delta rays have low kinetic energy, and they will be trapped between the foil and the Faraday-cup magnets, and should eventually be cleared by the blased foil. The medium-energy delta rays will be deflected into the Faraday cup, and the few (about $3 \times 10^{-5}$ per proton) high-energy delta rays that pass through the Faraday-cup magnets will be lost on the beam-pipe walls.

Figures 6 through 8 show some data from the various components of this system. In Fig. 6 we see some signals from the stripper foil with and without the bias turned on. Part (a) shows a strong signal that ramps up during the $500 \mu \mathrm{s}$ of beam injection. This is due to more and more circulating beam passing through the foll. During the $300 \mu \mathrm{s}$ of beam storage, the rate of electron emission decreases, and after extraction we see the signal drop off due to the RC time constant of the biasing circult. In part (b), where the foll is biased to $3 \mathrm{kV}$, the electron-emission rate continues to grow until extraction, but the overall signal amplitude is four times lower, indicating a reduced emission of electrons because they are now collected by the positively charged foil. The signal amplitude continues to drop as we raise the foll bias, but we have never observed the amplitude to drop completely to zero because of restrictions on the blas voltage due to breakdown effects. The highest we ever biased the foll was about $9 \mathrm{kV}$. In Fig. 7 we see some signals from the Faraday cup and screen. Part (a) shows the signals with the blases turned on but the bending magnets SRHMO1 and SRHMO2 turned off. These signals are due to low-energy electrons sucked out of the beam pipe, caused by SEM off the stripper foll and beam-pipe walls, and possibly a few delta rays and electrons from residual-gas ionization. In part (b) the bending magnets are turned on, thus steering the $430 \mathrm{keV}$ convoy electrons into the Faraday cup. The signal now has a rectangular shape to match the time structure of the injected beam, as expected. In Fig. 8 we show some clearing-electrode signals. Parts (a) and (b) show the positive electrode at 0 - and $10-\mathrm{kV}$ bias. As expected, turning up the bias voltage results in more electron collection. Parts (c) and (d) are the same thing but for the negative electrode. As expected, there is very little charge collected on this electrode since the collected particles are dominated by electrons. Part (e) shows an interesting phenomenon during the onset of beam instablity. Huge amounts of electrons are collected when the beam goes unstable. We see similar signals from the stripper foll, the Faraday cup and screen, and a pair of pinger electrodes located in section 3 of the ing. Three traces are shown to illustrate the pulse-to-pulse variations in the beam instability.

After commissioning the electron-clearing system this summer we found we can collect almost all the electrons created in section 0 . However, this raises the instability threshold of the coasting-beam instability by only about $20 \%$, and it increases the growth time of the bunched-beam instability by almost an order of magnitude but does not alter its threshold. This is a disappointing result for us but also an educational one. We now suspect there are other strong sources of electrons in the PSR, and we have initlated a new set of experiments to identify and characterize them.

\section{BEAM-IN-THE-GAP MONITOR}

By beam in the gap I mean beam that leaks into the gap between the front of the head and behind the tall of the single beam bunch in the PSR. The primary contribution to beam in the gap is space charge, which pushes protons out of the if bucket into the inter-bunch gap. We need to maintain this gap, nominally about $110 \mathrm{~ns}$ long, to allow time for the 


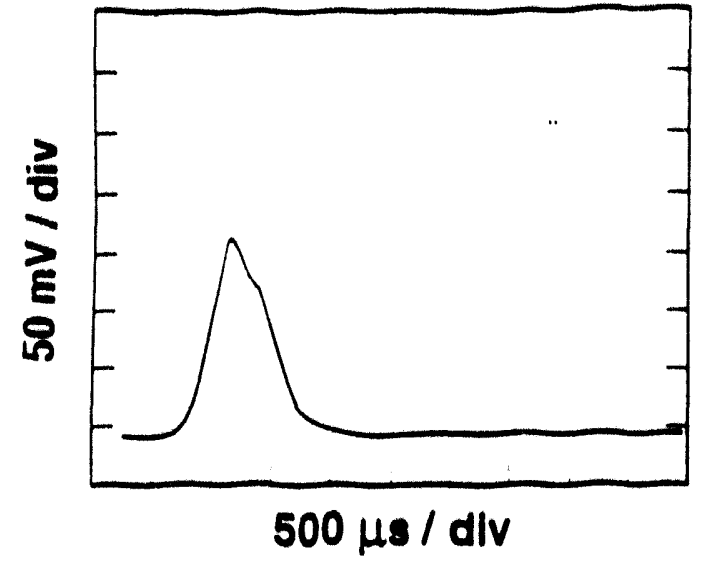

(a)

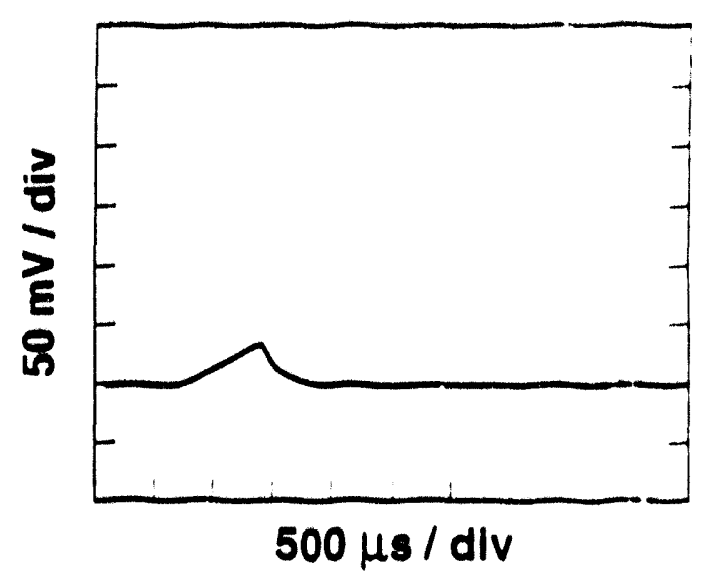

(b)

Fig. 6. Signals from the stripper foil, using capacitive coupling to the HV cable. There is a dc offset caused by the capacitive coupling circuit, so the baselines of the wavetorms should be considered meaningless. Part (a) is for $0 . V$ bias, and pant (b) is for $3-k V$ bias. Note that biasing the foil reduces the signal caused by electron emission from the foil. Beam parameters: injection time $=500 \mu \mathrm{s}$, storage time $=$ $300 \mu s$.

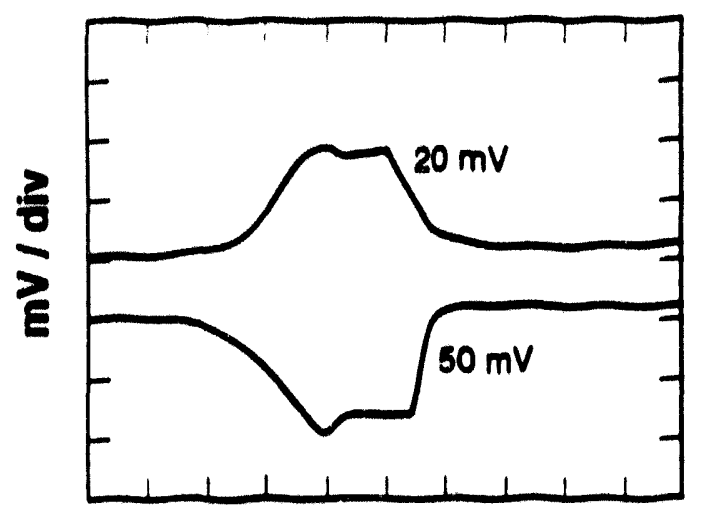

$200 \mu /$ dlv

(a)

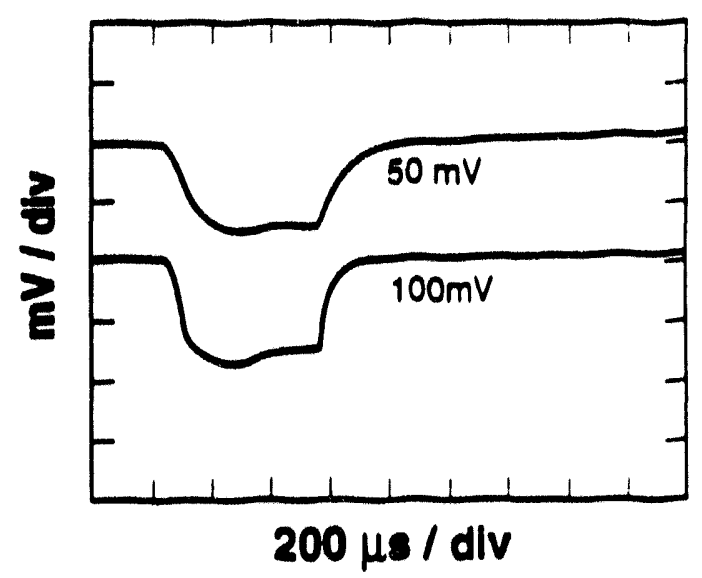

(b)

Fig. 7. Signals from the Faraday cup (bottom trace) and screen (top trace), using a low-pass fllter on an oscilloscope, with the power supply between the oscilloscope and the Faraday cup. The waveform baselines are arbitrary. In part (a) the SRHMO1 and SAHMO2 magnets are off, and in part (b) they are set to steer the convoy electrons into the Faraday cup. Faraday cup voltage $=200 \mathrm{~V}$, screen voltage $=100 \mathrm{~V}$. Beam paramoters: injection time $=500 \mu \mathrm{s}$, storage time $=300 \mathrm{\mu s}$. 


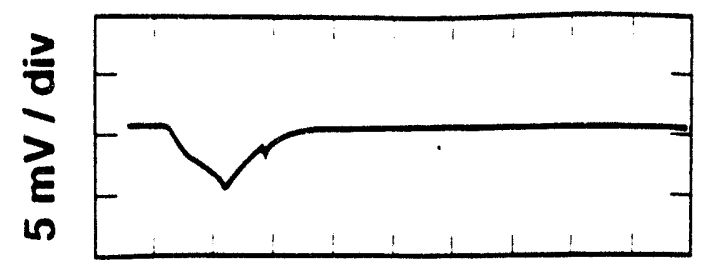

$500 \mu \mathrm{s} / \mathrm{div}$

(a)

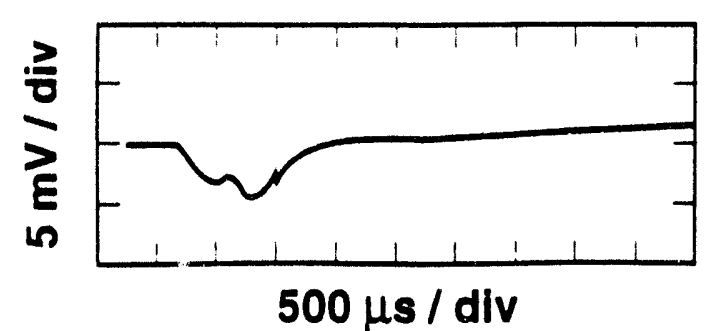

(c)

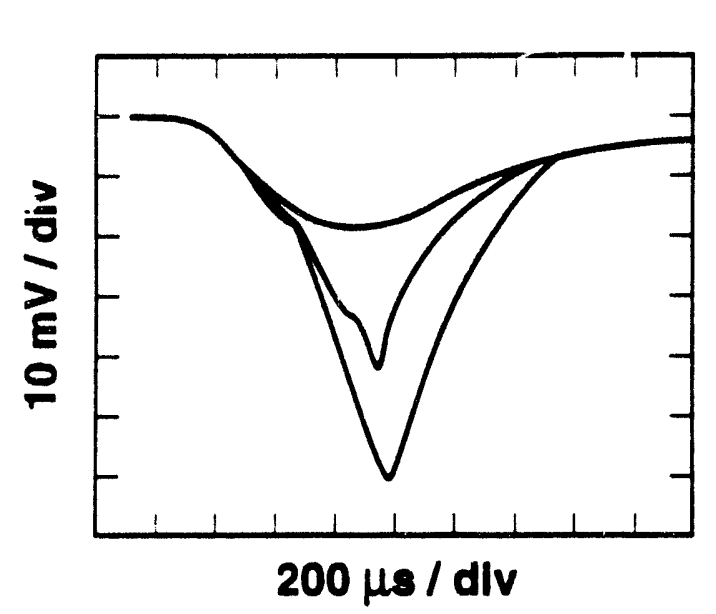

(ө)

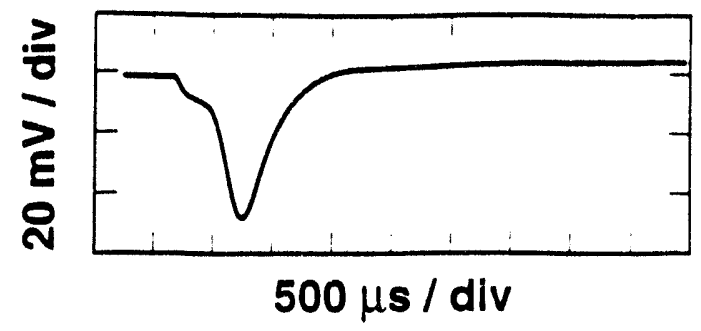

(b)

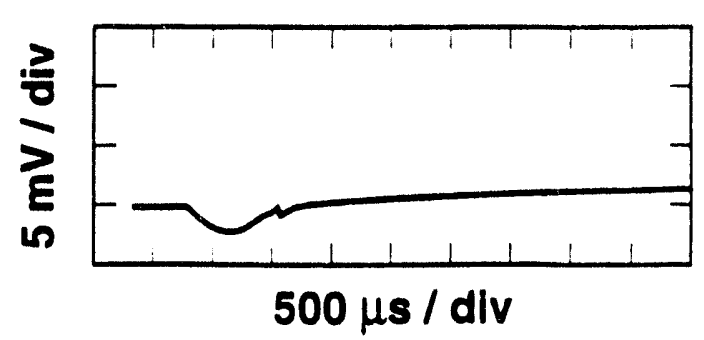

(d)

Fig. 8. Signals from the SRCOO2 (downstream) electrodes in the Electron-Clearing System, using capacittve coupling to the HV cable. There is a dc offset caused by the capacitive coupling circuit, so the baselines of the waveforms should be considered meaningless. Parts (a) and (b) show the positive electrode at 0 - and 10-kV blas, and parts (c) and (d) show the same thing but for the negative electrode. Beam parameters for parts (a) through (d): Injection time $=500 \mu \mathrm{s}$, storage time $=300$ us. Part (e) shows signals from the positive electrode of SRCQ02 during the coasting-beam instability. The electrodes are at $20 \mathrm{kV}$, the foil is at $6 \mathrm{kV}$, the SRHMO1 and SRHMO2 magnets are at $150 \mathrm{~A}$, the Faraday cup is at $200 \mathrm{~V}$, and the screen is at $100 \mathrm{~V}$. Three traces are shown to illustrate the pulse-topulse variations. Beam parameters: Injection time $=\mathbf{4 2 0} \mu \mathrm{s}$, storage time $=500$ $\mu s$, coasting beam (no bunching, injected beam fills the ring circumference). 
extraction kickers to turn on. Beam in the gap therefore has important implications to extraction beam loss. If there is beam in the gap, that beam will be sprayed onto the beam pipe when the extraction kickers turn on. Beam in the gap can also trap electrons that lead to an e-p instability, as opposed to a beam-free gap that will clear out the electrons as the gap sweeps around the ring by allowing the mutual repulsion of the electrons to cause them to fly away to the beam-pipe walls.

A good beam-in-the-gap monitor will accurately measure beam current more than four orders of magnitude below the peak beam current and have a response time of a couple of tens of nanoseconds. If the monitor is in the ring we would like a droop of less than $0.01 \% / \mathrm{ms}$, and if it is in the extraction line we would like a droop of less than $1 \% / \mu \mathrm{s}$. We thought of four ways to accomplish this measurement: with an electron beam probe, with a wide-band current monitor with very good low-frequency droop, by measuring secondary emission off the stripper foil, and with a current transformer in the extraction beam line. We ended up choosing the last option, but let me tell you why.

The idea behind the electron bea:n probe is to shoot an electron beam across the proton beam. ${ }^{8}$ The degree of vertical deflection is a measure of the proton current, and a pair of electrodes sweeps the electron beam horizontally to resolve the time structure. It's like running the proton beam line through the middle of an oscilloscope. After some investigation we decided this concept is not a good idea for several reasons: 1) an electron gun that would do the job (50 micron spot size at 1 meter, 2- to 20-keV energy, and $30 \mathrm{~mA} / \mathrm{cm}^{2}$ current density) is a very specialized device. The only one we found was a fieldemission gun. It has to be handled very carefully and it must be serviced at the factory, which is not equipped to deal with radioactive items. 2) A 2-keV electron beam is very sensitive to stray electromagnetic fields. For example, in a test at the factory, just putting a pair of pliers or scissors next to the beam pipe caused severe distortions for a 5-keV beam, even with a mu-metal shield inside the beam pipe. 3) The data would be difficult to interpret. We suspect there are a lot of electrons in the beam pipe, and these electrons would also deflect the electron beam. Additionally, a bunched proton beam will deflect the electron beam both vertically and horizontally, thus confusing the time-resolving axis.

We rejected the idea of a wide-band current monitor in the ring due to high cost and long development time. We rejected the idea of SEM off the stripper foil because of the high radiation fields, the low signal levels in a noisy environment, and because we expect the high-frequency response to be marginal due to the foil construction.

We settled on using a fast current transformer (FCT) in the extraction line. The idea here is to turn on the extraction kickers before the beam in the gap reaches the kicker electrodes, thus sending any beam in the gap down the extraction line and through the FCT (and also spraying full-intensity beam on the PSR beam-pipe walls). We purchased a custom-made FCT from Bergoz and installed it earller this year, about 47 meters downstream of the extraction kickers. Some of the specifications are shown in Table 3.

Table 5. Some specifications for the FCT.

\begin{tabular}{|l|l|l|}
\hline & Goal & Achieved \\
\hline Post-pulse settling time & $30 \mathrm{~ns}$ to $0.1 \%$ & $50 \mathrm{~ns}$ to $1 \%$ \\
\hline Rise time $(10 \%-90 \%)$ & $<5 \mathrm{~ns}$ & $17 \mathrm{~ns}$ \\
\hline Droop & $<1 \% / \mu \mathrm{s}$ & $0.6 \% / \mu \mathrm{s}$ over $4 \mu \mathrm{s}$ \\
\hline Transfer impedance & $>1.25 \Omega$ & $1.0 \Omega$ \\
\hline Inside diameter & $180 \mathrm{~mm}$ & $180 \mathrm{~mm}$ \\
\hline
\end{tabular}

We devoted a lot of effort to carefully shielding the FCT for noise. We stacked two tons of iron blocks for gamma-ray shielding, and we used 1.625-inch Heliax cabling inside a solid steel conduit to transmit the signal about 30 meters.

Although we missed our most important specification (the post-pulse settling time) by more than an order of magnitude, it turns out there is enough beam in the gap to measure it 
$-20,-15,-10,-5,0,5,10,15$, and $20 . n s$ changes in kicker timing

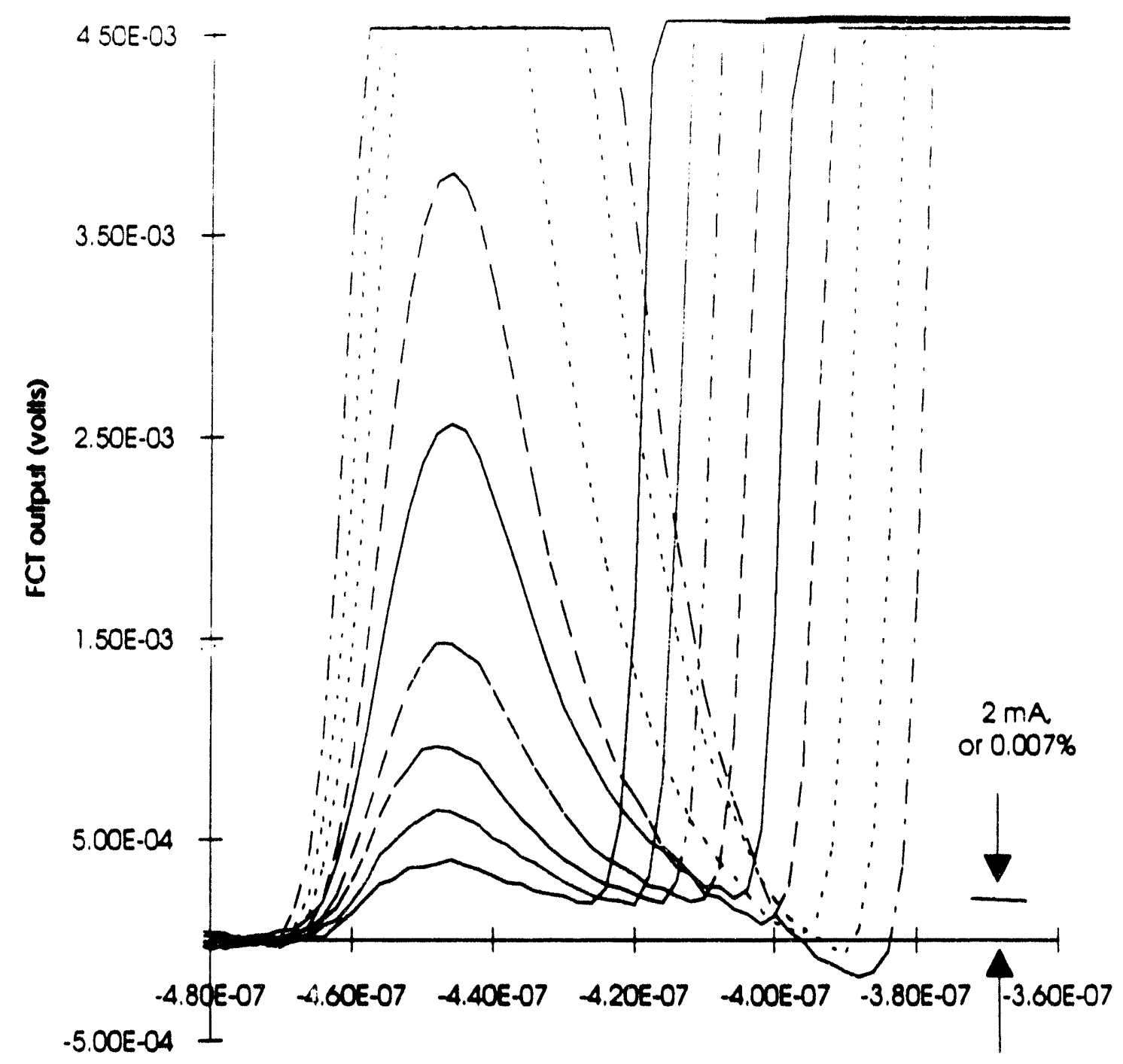

nmo (esconds)

Fig. 9. Beam-in-the-gap monitor signals taken during production conditions. The extraction kicker timing was changed by $-20,-15,-10,-5,0,5,10,15$, and $20 \mathrm{~ns}$. Beam parameters: Injection time $=525 \mu \mathrm{s}$, storage time $=10 \mu \mathrm{s}$. The oscilloscope was triggered on the extraction-kjcker waveform. 
FCT output for $100,200,300,500,600,700,725$ - $\mathrm{s}$ accumulation times

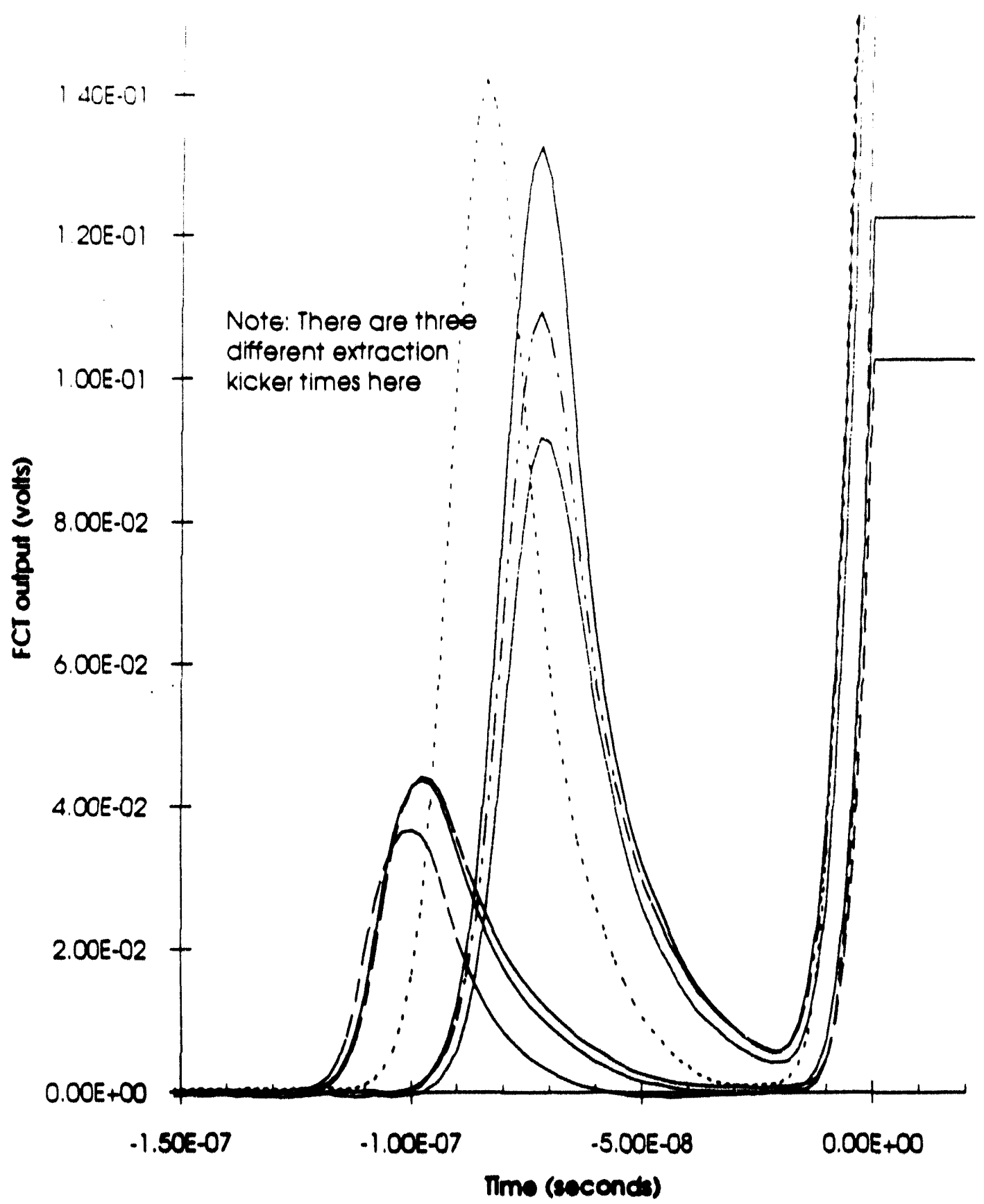

Fig. 10. Beam-in-the-gap monitor signals for seven diffierent injection times, and therefore seven different spece-charge fields. Note the gap fills in as more and more beam is injected into the ring. 
with this instrument. One can see from Fig. 9 that as one advances the kicker timing, one cuts into the tail of the beam more and more. In this figure, the trace with the lowestamplitude prepulse is from the kicker timing delayed $20 \mathrm{~ns}$. As we advance the kicker timing the prepulse amplitude increases, indicating that we are cutting into the tall of the beam pulse. The figure shows that if the amplitude of the prepulse is small enough, the FCT settles down enough to make the measurement. In this series of measurements, taken during production conditions, the beam in the gap dips down to about $2 \mathrm{~mA}$, or to about $0.007 \%$ of the peak beam intensity.

This instrument has also proven itself to be a useful diagnostic for setting the extraction-kicker timing, since it is more sensitive to the timing than our usual method of minimizing the extraction beam loss. From the above set of measurements we concluded that the initial kicker timing ( 0 ns on the figure) had been set too early, since the prepulse amplitude was not minimized. After we finished the measurements we left the timing delayed by $20 \mathrm{~ns}$, which resulted in a small imrovement in the operating conditions.

We can also vary the duration of the PSh i.ijection cycle and thus vary peak beam intensity and the space charge forces. Figures 10 and 11 show some preliminary data on how the beam-in-the-gap current changes as a function of the injection time. In Fig. 10 we see the FCT output plotted for seven different accumulation times, and therefore seven different peak currents in the ring. During these measurements we used three different extraction-kicker timings to keep the prepulse amplitude below acceptable limits. The figure shows that the gap current increases as we inject more and more beam into the ring. In Fig. 11 we plot the beam in the gap as a function of the accumulation time, expressed as a percentage of the peak beam current. In this figure there are three groups of data points because of the three different sets of extraction-kicker timings and therefore three different manifestations of the droop. We see from this figure that as we increase the beam current, and therefore the space-charge flelds in the ring, the beam in the gap increases correspondingly.

\section{SUMMARY AND CONCLUSIONS}

We have discussed some of the constraints that high intensity accelerators and storage rings place on beam-diagnostic instruments. Most of these are related to beam halos and beam space charge. We alsc discussed in some detall three diagnostic systems in use at the PSR that are related to high-intensity issues - the loss-monitor system, the electron-clearing system, and a fast-current transformer for measuring beam in the gap.

Wo have a wide variety of beam-loss-monitoring systems at LAMPF because beam loss is 80 important to us - for example, it prevents the PSR from reaching its design goal of $100 \mu A$ average current. We discussed two of our loss-monitor systems - the PSR perimeter system with twenty detectors that can measure absolute beam loss that is a fraction of a percent of the total beam current, and the fail-safe ion-chamber system that is a necessary ingredient in our personnel-protection system.

Next we moved on to the electron-clearing system, which we bullt to remove the electrons from section 0 of the PSR. Although we succeeded in clearing out these electrons we did not substantlally improve the instability threshold. However, new experiments suggest other sources of electrons, and we are now working to identlfy and characterize them.

The final subject was the beam-in-the-gap monitor. We installed a custom-made fastcurrent transformer in the PSR extraction line. By firing the extraction kickers early beam in the gap is directed into the extraction line to the FCT. Preliminary results show that for production conditions the beam-in-the-gap current is $0.007 \%$ of the peak beam current.

I hope these three examples of beam-diagnostic systems at PSR have highlighted some of the interesting challenges available at high-intensity facilities. 
PRELIMINARY DATA

Beam in the gop as a traction of peak current

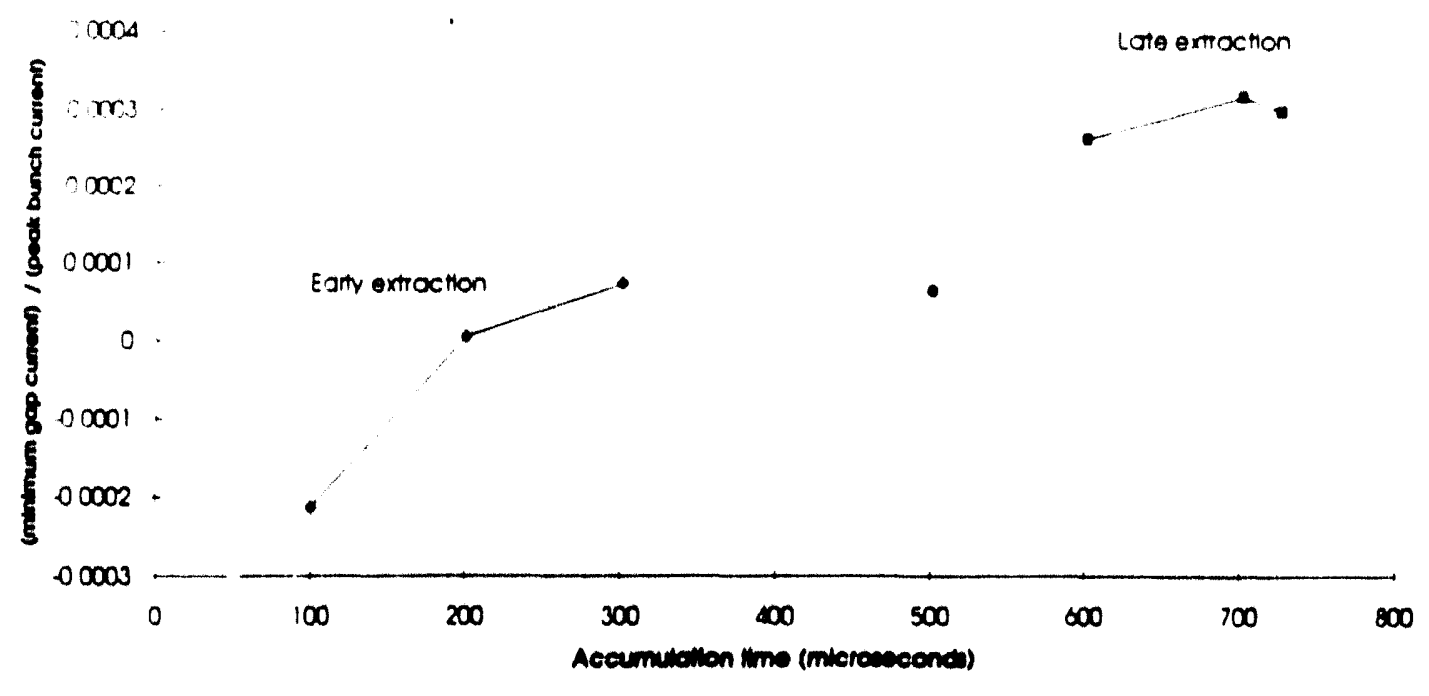

Fig. 11. Minimum beam-in-the-gap current as a function of injection time.

1 R. Jameson, "Halo Formation in Linace," in Brocenedinge of the Workshop on Accalerators for Future Soallation Nentron Souren, Feb. 16-20, 1993. Also Los Alamos National Laboratory report LA-UA-93-1360.

2 M.A. Plum ot al., "Fall-Safo lon Chamber Errant Beam Dotector Tailored for Personnel

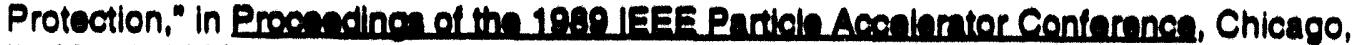
IL, March 1989, pg. 1586, and Los Alamos National Laboratory report LA.UR-89-1183.

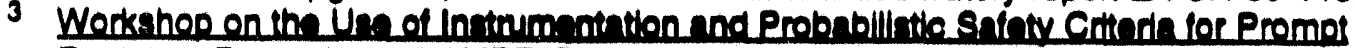
Badiation Protexition nt L AMPE, Decombor 9-11, 1991, Los Alamos, NM, Los Alamos National Laborntory report LA-UR-92-300.

4 Ibid.

5 M.A. Plum and D. Brown, Reapones of Air-Filled Ion Chambers to High-Intensity

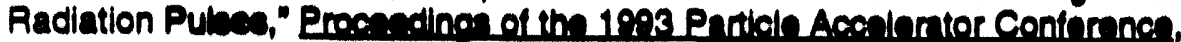
Washington, D.C., May 17-20, 1993. Aleo Los Alamos National Laboratory roport LA.UR. 92-4066.

B D. Nouffer of al., "Obaervations of a fast transvorse inatability in the PSP," Nucl. Instrum. Mothods AB21, (1) 1002.

7 F. Saull, "Principles of Operation of Multwwire Proportional and Dith Chambers," CERN 77-09.

8 J.A. Pasour and M.T. Nog, Rev. Sot. Inctrum. 02, 3027 (1902). 


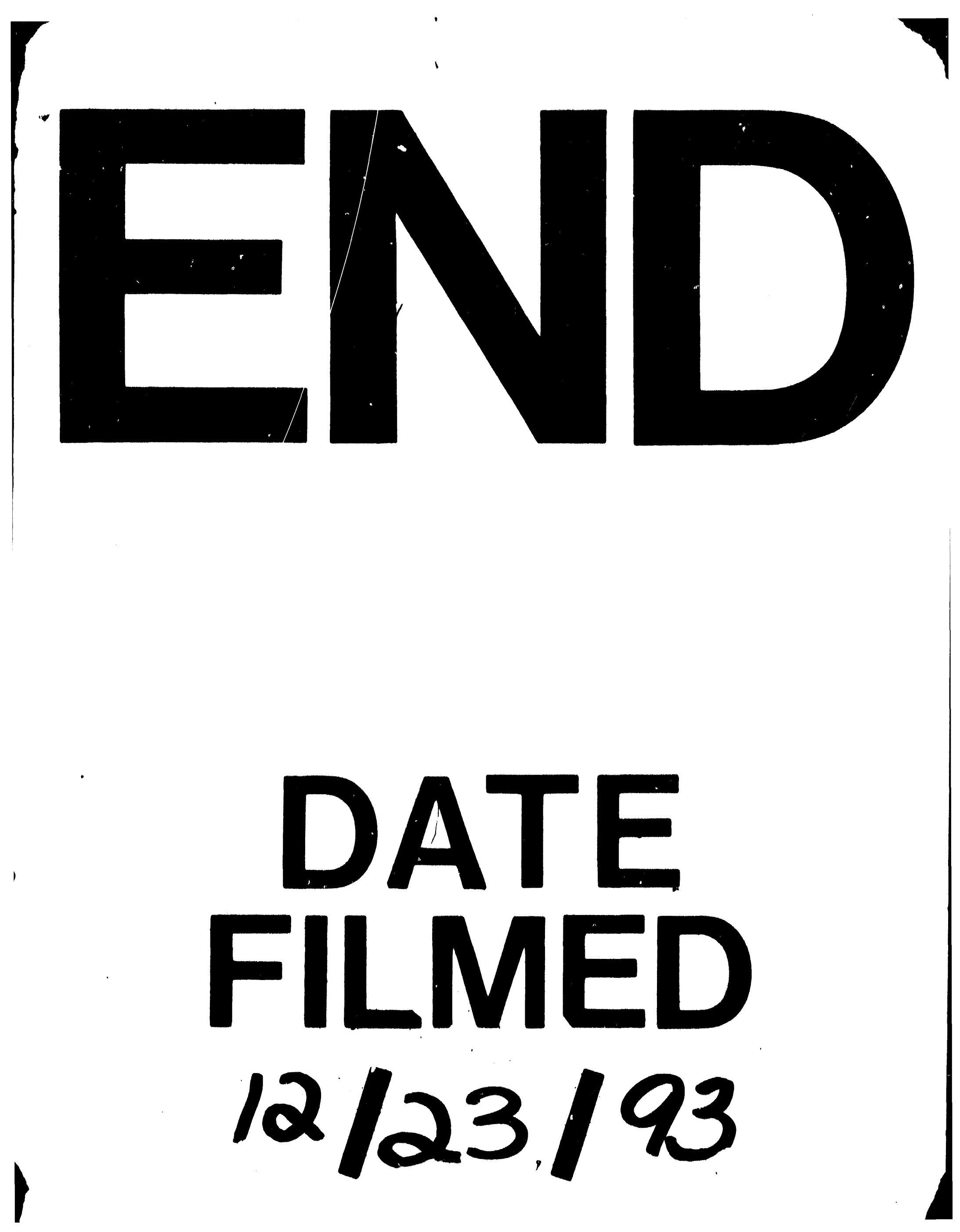


